\title{
Recovery - warum nicht?
}

\author{
Recovery - Why Not?
}

Autor

Institut
Michaela Amering

Universitätsklinik für Psychiatrie und Psychotherapie, Abt. für Sozialpsychiatrie

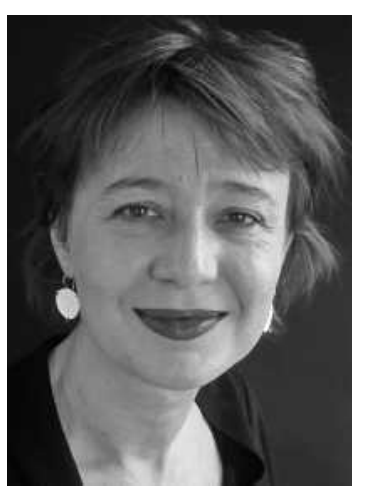

Univ.-Prof. Dr. Michaela Amering

\section{Bedeutung \\ $\nabla$}

Recovery beschreibt in seiner traditionellen Bedeutung - Genesung, Wiederherstellung, Gesundung - klassische Ziele jeder therapeutischen Intervention. Als Ron Coleman Ende der 90er-Jahre Recovery als eine fremdartige Idee - „an alien concept" - bezeichnete und ausdrückte, dass PatientInnen und professionelle HelferInnen offenbar allesamt vergessen hatten, dass man sich von Schizophrenie wieder erholen kann [1], hat wohl auch er kaum damit gerechnet, dass Recovery in den kommenden Jahren ganz neu "in Mode“ kommen würde. Colemans eigene Geschichte illustriert, dass auch nach langjährigen katastrophalen Behandlungsverläufen Gesundung möglich ist, zeigt aber auch, wie sehr sich die früh widerlegte Kraepelin'sche These von der Unheilbarkeit in falschen negativen prognostischen Einschätzungen aller Beteiligten niederschlägt.

2005 wurden für die Schizophrenie Remissionskriterien formuliert [2]. Davon erwartet man sich nicht nur eine Korrektur der Einschätzung der Prognose und klare Vorteile im Messen und Vergleichen von Verläufen und Interventionen, sondern auch, dass die Erwartungen erhöht und die therapeutischen Bemühungen verstärkt werden. Ebenso wie der US-amerikanische Vorstoß weist auch die rasche und affirmative europäische Antwort [3] klar darauf hin, dass Recovery jedoch noch etwas anderes und mehr bedeutet als Remission im Sinne des Abklingens von Symptomen und der Steigerung der Funktionsfähigkeit.

Pat Deegans [4] Analysen dazu, wie sie und andere Betroffene aus den Beschränkungen der Patientenrolle heraus zu einem selbstbestimmten Leben gefunden haben, zeigen klar, worum es geht, wenn von Recovery die Rede ist. Es geht nicht um eine Rückkehr zu einem Zustand vor der Erkrankung, sondern vielmehr um ein Wachstum und eine Entwicklung, die es ermögli- chen, die persönlichen, sozialen und gesellschaftlichen Folgen einer psychischen Erkrankung zu überwinden. Das Leben soll sich an den persönlichen Werten und Zielen orientieren und kann zufriedenstellend und hoffnungsvoll verlaufen, auch wenn Symptome und Behinderungen über längere Zeit bestehen. Wesentlich ist, Selbstwert und Selbstachtung unabhängig von der PatientInnenrolle zu erleben und zu wissen, dass eine psychiatrische Diagnose nicht die Entwicklung von Resilienz verhindert [5].

Die Recovery-ProponentInnen aus der Betroffenenbewegung machen klar, dass Recovery ein individueller Prozess ist, bei dem es um persönliche Werte und Lebensziele geht im Gegensatz zu statistisch erfassbaren „objektiven“ Kriterien. Häufig werden in der wissenschaftlichen Recovery-Literatur grob zwei Definitionen unterschieden [6]:

1. die Reduktion von Symptomen und Behinderungen und

2. die Erfolge und Bemühungen mit oder trotz psychischer Probleme, Behinderungen, Anfälligkeiten oder Besonderheiten ein selbstbestimmtes und ausgefülltes Leben zu führen. Die erste Definition wird dem medizinischen Bereich zugerechnet und als „klinisch“ im Unterschied zur zweiten „rehabilitativen“ Definition [7] oder als basierend auf den Konzepten der Versorgung - „service-based“ - bzw. basierend auf den Konzepten der Betroffenen - „user-based“ [8] - benannt. Es wird angezweifelt, ob es zu einer Integration dieser beiden unterschiedlichen Perspektiven kommen muss. Beide können komplementär Bedeutung haben und unterschiedlichen Personengruppen und Absichten dienen [7]. 


\section{Entwicklung \\ $\nabla$}

Recovery-Konzepte sind keineswegs auf Schizophrenie beschränkt. Recovery ist heute in vielen Ländern gesundheitspolitische Vorgabe für den Bereich der psychiatrischen Versorgung und Gesundheitsförderung. Drei wesentliche Punkte, die Recovery derzeit im Wege stehen, hat eine Gesundheitskommission der Regierung der USA in den Vordergrund gestellt [9]:

- das Stigma, das psychische Erkrankungen noch immer umgibt;

- die weiter vorherrschenden Ungerechtigkeiten und Ungleichbehandlung im Versicherungssystem;

- das fragmentierte psychiatrische Versorgungssystem.

Diese drei Punkte machen deutlich, dass bei allen Unterschieden zwischen Europa und den USA große Probleme identisch und schwierig zu überwinden sind.

Mit Blick auf die Hauptziele der Psychiatriereform - Schließung der alten Anstalten, Entwicklung gemeindenaher Versorgungssysteme, Integration von psychiatrischen Angeboten in den allgemeinen Gesundheitsbereich, Integration von sozialen und Gesundheitsleistungen - ist festzustellen, dass sie in westeuropäischen Ländern zu etwa 30-70\% umgesetzt sind [10]. Hauptsächlichen Aufholbedarf orten Becker u. Vazquez-Barquero [11] beim Zugang zum Arbeitsmarkt, der Integration von psychiatrischen Angeboten in den allgemeinmedizinischen Bereich und der Unterstützung von Angehörigen und Freunden - alles für Recovery relevante Bereiche. Internationale Zahlen zeigen, dass weniger als $25 \%$ aller PatientInnen mit schweren psychiatrischen Erkrankungen diejenigen Behandlungen erhalten, die umfassend evidenzbasierten Vorschlägen entsprechen [12].

Viele Recovery-Entwicklungen finden außerhalb der Psychiatrie statt [13]. Entscheidend wichtig sind häufig ganz spezifische individuell wirksame Ansätze ohne Anspruch darauf, dass genau dasselbe auch den meisten anderen Menschen helfen könnte [14]. Dabei finden Betroffene meist zu wenig Unterstützung durch professionelle HelferInnen.

Das mag sich ändern, wenn sich die Rollenverteilung zwischen dem Hilfesystem und seinen NutzerInnen verändert. PatientInnen als ExpertInnen in eigener Sache, Selbstmanagement länger dauernder Erkrankungen, partizipative Entscheidungsfindung, Behandlungsvereinbarungen, Vorausverfügungen und Trialog und Psychoseseminar sind Entwicklungen, die Ausdruck einer neuen Rollenverteilung zwischen KlinikerInnen und PatientInnen sind. PatientInnen haben eine aktive KonsumentInnenrolle in einem Dienstleistungsverhältnis übernommen [15]. Die Rolle der PsychiaterInnen erweitert sich in Richtung Coaching und Mentoring und neue Fähigkeiten zum partnerschaftlichen Umgang mit Entscheidungen und zur Unterstützung von Selbstbestimmung werden unterrichtet. Meine Forschungstätigkeit zu psychiatrischen Vorausverfügungen [16] in New York hat gezeigt, dass auch PatientInnen mit langen Geschichten von Zwangsbehandlungen und Verletzungen und Enttäuschungen durch das psychiatrische Versorgungssystem bereit sind mit großer Verantwortung und Umsicht ihre Selbstbestimmung zur Verbesserung ihrer Behandlung in Akutsituationen wahrzunehmen.

\section{Verantwortung \\ $\nabla$}

Professionelle HelferInnen sehen die Haupthindernisse für eine Entwicklung zu recovery-orientierten Angeboten in Fragen ihrer Verantwortung und dem stetig verspürten Mangel an Ressourcen und der Verpflichtung therapeutische Interventionen zu setzen, auf deren Effekte - oder deren unerwartete Unwirksamkeiten - man dann Recovery-Erfolge oder Misserfolge zurückführen kann. Eine Annäherung der Psychiatrie an andere Bereiche der Medizin, in denen Wahlfreiheit und Selbstbestimmung der PatientInnen nicht traditionell infrage steht, könnte hier als Orientierung dienen [17]. Ebenso wie verbesserter Schutz vor Diskriminierung könnte dazu eine Gesetzgebung, die Zwangsmaßnahmen nicht auf der Basis von psychiatrischen Diagnosen, sondern unabhängig davon auf Basis von Entscheidungsfähigkeit und gemeinsam für alle Personen im gesamten Gesundheitswesen regelt, ein Fortschritt sein [18].

Mike Slades [19] Beispiel von einer notwendigen Debatte um die Empfehlenswürdigkeit einer Intervention, von der wissenschaftlich erwiesen ist, dass sie Symptome reduziert, aber Abhängigkeit und Hoffnungslosigkeit fördert, deutet an was Recovery-Forschung leisten muss, damit das Konzept bestehen bleiben und Veränderungen bewirken kann. Um zu erforschen was Hilfeangebote tatsächlich förderlich für Recovery macht, muss es möglich werden, nicht nur zu beschreiben, was Einrichtungen und HelferInnen tun, sondern auch wie sie es tun, also eine Erfassung von Haltungen und Werten, die in therapeutischen Kontakten und Interventionen zum Ausdruck kommen. Um Ergebnisse von Recovery-Prozessen zu messen, müssen Ergebnisvariablen definiert werden, die auch subjektive, an individuellen Werten und Zielen orientierte Ergebnisse messbar machen. Eine günstige Voraussetzung dafür ist der Ausbau von Forschungskooperationen zwischen Betroffenen und ForscherInnen sowie zwischen ForscherInnen, die eigene Erfahrungen mit psychischen Störungen gemacht haben und solchen ohne entsprechende Erfahrungen [20]. Eine andere Möglichkeit ist die Einbeziehung von Personen, die selbst Recovery erfahren haben, in die Planung, Durchführung und Evaluation von Hilfeangeboten [21]. Wesentliche Fragen betreffen auch die Aus- und Weiterbildung in recovery-orientiertem Arbeiten. Auch hier bietet sich klar der Einsatz von Personen mit eigener Recovery-Erfahrung als Bereicherung an. Die Forderung nach einer Evidenzbasis, die unterschiedliche Perspektiven integrieren kann, geht Hand in Hand mit der Forderung nach der kompetenten Anwendung und Mischung unterschiedlicher Forschungsmethoden und partizipativen Prozessen zur Erstellung von Richtlinien [22].

Recovery leuchtet ein, entspricht dem „common sense“. Das ist viel verlangt für die Psychiatrie. Aber so wünsche ich sie mir. Und weil es gerade so viele Stimmen dafür gibt und so viel klugen Einsatz und hochkarätiges Engagement, könnte sich doch glatt was tun in dieser Richtung. 


\section{Literatur}

1 Coleman R. Recovery - an alien concept. Gloucester, UK: Handsell Publishing, 1999

2 Andreasen NC, Carpenter WT, Kane JM, Lasser RA, Marder SR, Weinberger $D R$. Remission in schizophrenia: proposed criteria and rationale for consensus. Am J Psychiatry 2005; 162: 441 - 449

3 van Os J, Burns T, Cavallaro R, Leucht S, Peuskens J, Helldin L, Bernardo $M$, Arango C, Fleischhacker W, Lachaux B, Kane JM. Standardized remission criteria in schizophrenia. Acta Psychiat Scand 2006; 113: 91 - 95

4 Deegan P. Recovery as a journey of the heart. In: Davidson L, Harding C, Spaniol L (eds): Recovery from severe mental illnesses: research evidence and implications for practice. Vol 1. Center for Psychiatric Rehabilitation, Trustees of Boston University, 2005: 57-68

5 Glover $H$. Recovery based service delivery: are we ready to transform the words into a paradigm shift? AeJAMH 2005; 4 (3): 1 -4

6 Schrank B, Amering M. Recovery in der Psychiatrie. Neuropsychiatrie 2007; $21: 45-50$

7 Davidson L, Lawless MS, Leary F. Concepts of recovery: competing or complementary? Curr Opin Psychiatry 2005; 18: 664-667

8 Schrank B, Slade M. Recovery in Psychiatry. Psychiatric Bulletin 2007; 31: $321-325$

9 Amering M, Schmolke M. Recovery. Das Ende der Unheilbarkeit. Bonn: Psychiatrie-Verlag, 2007

10 Becker T, Kilian R. Psychiatric services for people with severe mental illness across western Europe: what can be generalized from current knowledge about differences in provision, costs and outcomes of mental health care? Acta Psychiat Scand 2006; 113 (Suppl 429): 9-16

11 Becker T, Vatquez-Barquero JL. The European perspective of psychiatric reform. Acta Psychiatr Scand 2001; 104 (Suppl 410): 8- 14
12 Anthony W, Rogers ES, Farkas M. Research on evidence-based practices: future directions in an era of recovery. Community Ment Hlt J 2003; 39: $101-114$

13 Lehmann P, Stastny P. Statt Psychiatrie. Berlin: Antipsychiatrieverlag, 2007

14 Frese FJ, Stanley J, Kress K, Vogel-Scibilia S. Integrating evidence-based practices and the recovery model. Psychiatr Serv 2001; 52: 1462 1468

15 Priebe S. Zukunft psychiatrischer Versorgung - Träume und Alpträume. Psychiat Prax 2003; 30 (Suppl 1): 48 -53

16 Amering M, Stastny P, Hopper K. Psychiatric advance directives: qualitative study of informed deliberations by mental health service users. Br J Psychiatry 2005; 186: 247-252

17 Davidson L, O'Connell M, Tondora J, Styron T, Kangas K. The Top Ten Concerns About Recovery Encountered in Mental Health System Transformation. Psychiatr Serv 2006; 57: 640-645

18 Dawson J, Szmukler G. Fusion of mental health and incapacity legislation. Br J Psychiatry 2006; 188: 504-509

19 Slade M. Recovery, psychosis and psychiatry: research is better than rhetoric. Acta Psychiatr Scand 2007; 116: 81 - 83

$20 \mathrm{Krumm}$ S, Becker T. Der Einbezug von Nutzern psychiatrischer Angebote in die psychiatrische Versorgungsforschung. Psychiat Prax 2006; 33: $59-66$

21 Sibitz I, Swoboda H, Schrank B, Priebe S, Amering M. Einbezug von Betroffenen in Therapie- und Versorgungsentscheidungen: professionelle HelferInnen zeigen sich optimistisch. Psychiat Prax 2007 Nov 6; Epub ahead of print

22 Rose $D$, Thornicroft $G$, Slade M. Who decides what evidence is? Developing a multiple perspectives paradigm in mental health. Acta Psychiatr Scand 2006; 113 (Suppl 429): 109-114 